LA-9094-MS

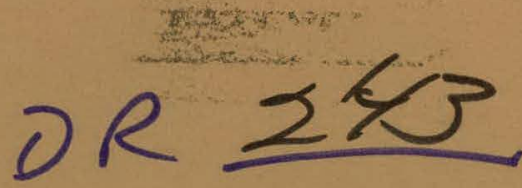

\title{
Analysis of Ventilation Systems Subjected to Explosive Transients \\ Far-Field Analysis
}

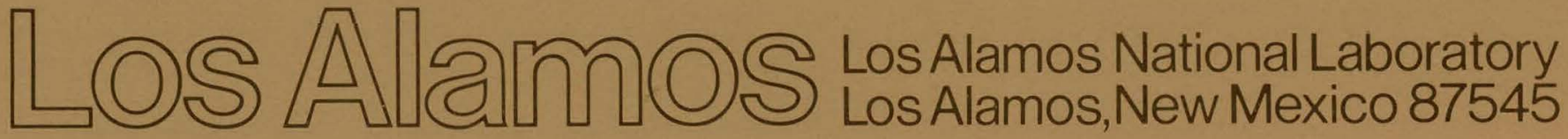




\section{DISCLAIMER}

This report was prepared as an account of work sponsored by an agency of the United States Government. Neither the United States Government nor any agency Thereof, nor any of their employees, makes any warranty, express or implied, or assumes any legal liability or responsibility for the accuracy, completeness, or usefulness of any information, apparatus, product, or process disclosed, or represents that its use would not infringe privately owned rights. Reference herein to any specific commercial product, process, or service by trade name, trademark, manufacturer, or otherwise does not necessarily constitute or imply its endorsement, recommendation, or favoring by the United States Government or any agency thereof. The views and opinions of authors expressed herein do not necessarily state or reflect those of the United States Government or any agency thereof. 


\section{DISCLAIMER}

Portions of this document may be illegible in electronic image products. Images are produced from the best available original document. 
This work was supported by the US Department of Energy, Division of Operational and Environmental Safety.

This report was prepared as an account of work sponsored by an agency of the United States Government. Neither the United States Government nor any agency thereof, nor any of their employees, makes any warranty, express or implied, or assumes any legal liability or responsibility for the accuracy, completeness, or usefulness of any information, apparatus, product, or process disclosed, or represents that its use would not infringe privately owned rights. References herein to any specific commercial product, process, or service by trade name, trademark, manufacturer, or otherwise, does not necessarily constitute or imply its endorsement, recommendation, or favoring by the United States Government or any agency thereof. The views and opinions of authors expressed herein do not necessarily state or reflect those of the United States Government or any agency thereof. 
LA $--9094-M S$

LA-9094-MS

UC-38

Issued: November 1981

DE82 010025

\title{
Analysis of Ventilation Systems \\ Subjected to Explosive Transients
}

Far-Field Analysis

\author{
P. K. Tang \\ R. W. Andrae \\ J. W. Bolstad \\ K. H. Duerre \\ W. S. Gregory
}

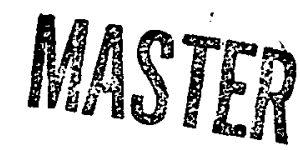

This book was prepared as an account of work sponsored by an ageney of the United States Government.

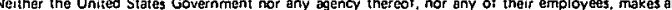
warranty, express or implied. or assumes any legal liability of responsibility for the accuracy. compiezeness, or useiutness of any iniormation, apparotus, product. of process clisclosed. represents that lis use would not intringe privately owned rights. Relerence herain to any specific

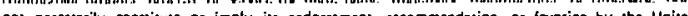

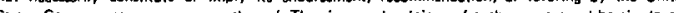
Los Alamos,New Mexico 87545 


\section{ANALYSIS OF VENTILATION SYSTEMS SUBJECTED \\ TO EXPLOSIVE TRANSIENTS \\ Far-Field Analysis}

by

P. K. Tang, R. W. Andrae, J. W. Bolstad, K. H. Duerre, W. S. Gregory

\section{ABSTRACT}

This report outlines progress in developing a far-field explosion simulation computer code. The term far-field implies that this computer code is suitable for modeling explosive transients in ventilation systems that are far removed from the explosive event and are rather insensitive to the particular characteristics of the explosive event. This type of analysis is useful when little detailed information is available and the explosive event is described parametrically. The code retains all the features of the TVENT code and allows completely compressible flow with inertia and choking effects. Problems that illustrate the capabilities and limitations of the code are described.

\section{INTRODUCTION}

This report discusses development of a computer code that will predict flow dynamics within structures subjected to internal explosions. We believe that this analysis tool will aid analyses of nuclear and nonnuclear Department of Energy facilities. The computer code places emphasis on flow dynamics within a complex interconnected facility ventilation system, but it is also applicable to other airflow pathways within structures. 
The report describes the evolution of the explosion code from the TVENT code. ${ }^{1}$ We will review our approach to the development of an explosion computer code and outline the desirable features of TVENT that are retained. We also describe the mass and energy equations added to the code to describe compressible fluid dynamics resulting from explosive events. The incompressible duct flow requirement in TVENT is eliminated by using a form of the momentum equation that includes both inertia and choking effects. This equation will be discussed in considerable detail because it forms the basis for the far-field version of the explosion code. Several examples are used to illustrate the capability and limitations of this far-field version.

\section{BACKGROUND}

\section{A. Approach}

Our general approach in developing a computer code to model explosive transients within structures was described in an earlier report. ${ }^{2}$ In this report we will review our approach to the explosion computer code, particularly with respect to the relationship between the TVENT. formulation and the first far-field version. The organization of the explosion code is shown in Fig. 1 .

The explosion code evolution from the TVENT code with the necessary modifications is also shown in Fig. 1 . When the explosive event is considered, we subdivide the code for near-field or far-field analysis. The distinction. between near-field and far-field regions is illustrated in a different manner, in Fig. 2, which shows a ventilation system that has been subdivided into near and far-field regions. A near-field region surrounds the explosive event location where the phenomenology is sensitive to the detailed hydrodynamics of the explosion. Far-field regions are those farther from the explosive event where the flow dynamics are relatively insensitive to the detailed characteristics of the explosive event.

In any case, as shown in Fig. 1, the user must decide whether to make a near or a far-field analysis. Results of a near-field analysis will provide the driving potential for analyses in the far-field region. However, in many cases the near-field analysis can be bypassed in favor of a far-field analysis only. This decision will probably be based on (1) the availability of 


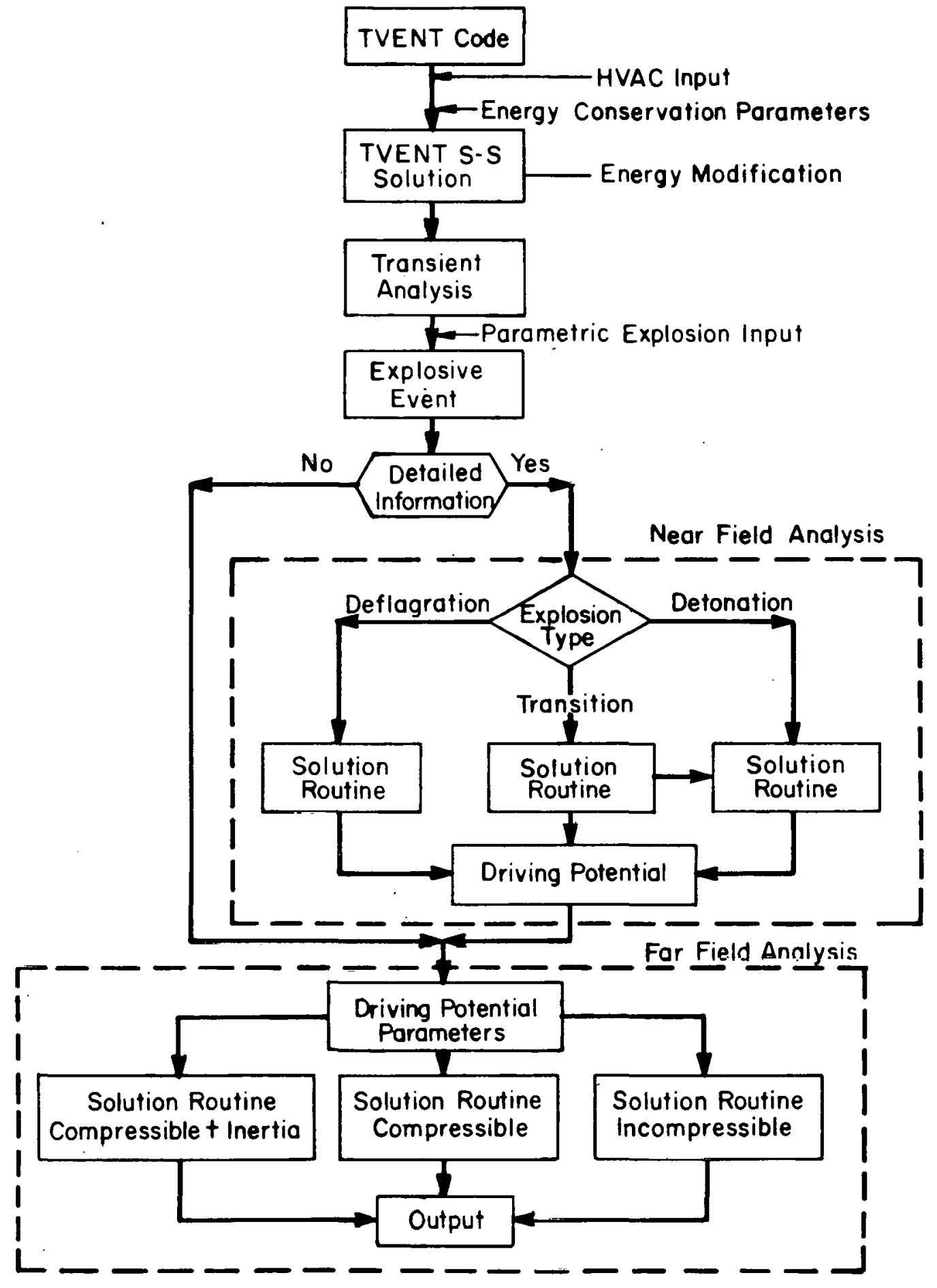

Fig. 1. Explosion code organization. 
detailed information describing the characteristics of the explosive event and the system geometry, and (2) the desirability of performing a highly detailed analysis before undertaking a less detailed or first-order analysis.

We have chosen to develop far-field analysis methods first for the following reasons.

- First-order results can be obtained with this version of the code. (In many cases this may be the only analysis required.)

- It is a logical and simple next step in development from the TVENT code.

- An early first version of the explosion code can be released.

\section{B. TVENT Basis}

In developing the new code, we intend to retain.as much of the original TVENT code as possible because we will use TVENT as a foundation on which to build the explosion code. TVENT features to be retained are the code superstructure, its portability, and the numerical approach to solving the governing equations.

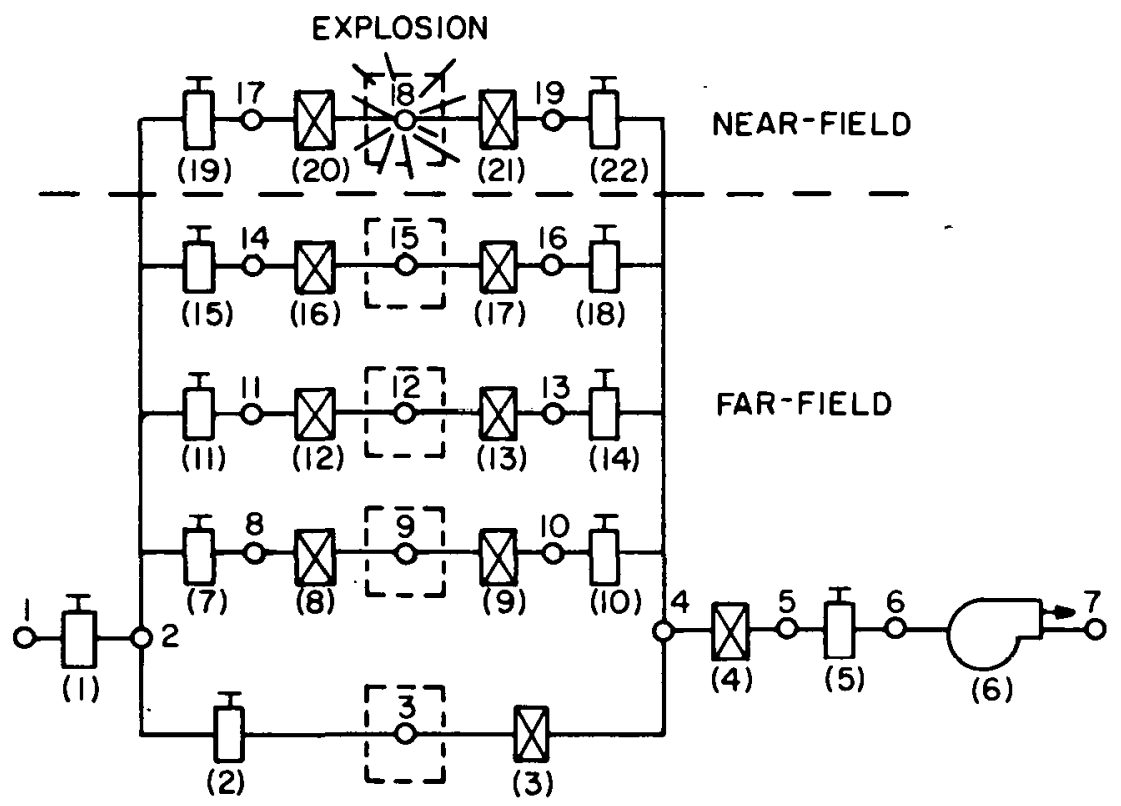

Fig. 2. Near- and far-field regions in a ventilation system. 
The TVENT superstructure will allow the explosion code to keep the same general organization. The input and the output routines will be retained because they are particularly suited for the heating, ventilating, and air conditioning (HVAC) designer and analyst. The type of output (line printer) found in TVENT will still be used, which will also help to retain portability. TVENT has been run on several types of computers, and we intend that the explosion code have this feature. This may be difficult to achieve because additional equations must be solved for explosive phenomena, which increases computer storage and running time.

The steady-state analysis capability of TVENT will also be preserved in the explosion code. However, special provisions for solving the full, set of equations, particularly the equation of state, must be made because steadystate conditions mean that air density and temperature variations are small. Perhaps the most difficult feature to retain from TVENT is the numerical procedure for solving the governing fluid dynamic equations.

The lumped-parameter method is the basic TVENT formulation that describes a ventilation system. No spatial distribution of parameters is considered in this approach. Network theory, using the lumped-parameter method, includes a number of system elements, called branches, joined at certain points, called nodes. Ventilation system components that exhibit resistance, such as dampers, filters, and blowers, are located within the branches of the system. The ductwork of a ventilation system is considered a resistive element because of the frictional resistance to fluid flow.

The connection points (nodes) of the system elements or branches are at the upstream and downstream ends of the branches. Components that have larger volumes, such as rooms, gloveboxes, and plenums, are located at nodal points. Therefore, a node may possess some volume or capacitance where fluid storage or compressibility may be accounted for.

Governing equations in TVENT require that the continuity equation be satisfied at every node and that a pressure-flow equation be satisfied for each element or branch. Variations in the node equations depend on whether the node represents a volume or a simple connection of two branches. If the node represents a finite volume, the equation of state for a perfect gas must be satisfied. This variation also exists for the branches, depending on whether the branch is simply a duct or contains a filter, blower, or damper. 
The relationship between pressure and flow for the elements is nonlinear and is written in general form

$$
Q=R\left(p_{i}-p_{j}\right)^{N}
$$

The volumetric flow rate is represented by $Q$, resistance by $R$, pressures at the ends of a branch by $p_{j}$ and $p_{j}$, and an arbitrary exponent by $N$. This numerical scheme uses a perturbation technique coupled with a Taylor series expansion of Eq. (1). For a small perturbation ( $\widetilde{\Delta p}$ ) the correct value for pressure $p_{j}$ is

$$
p_{j}=\widetilde{p}_{j}+\widetilde{\Delta p}
$$

where the sign indicates a temporary value. Substituting Eq. (2) in Eq. (1) gives

$$
Q=R\left(p_{i}-\tilde{p}_{j}-\widetilde{\Delta p}\right)^{N}
$$

Using a Taylor series expansion of Eq. (3) gives a linear relationship between flow and pressure as

$$
Q=\tilde{A}-\tilde{C} \tilde{\Delta P},
$$

where $\tilde{A}$ and $\tilde{C}$ are temporary iterative values based upon previous values of pressure. The flow-pressure relationship in the branches for the ducts, dampers, filters, and blowers can all be formulated as Eq. (4). Now all flows must satisfy the continuity equation at the nodal points. Summing all flows at a nodal point allows us to solve for the perturbation pressure $\widetilde{\Delta p}$. The numerical process then iterates through a set of equations for $\tilde{\Delta p}$ until that value is very small. Then we say that the numerical process has converged and all the governing equations are satisfied. This numerical scheme is a fully implicit iterative process.

Retaining the TVENT numerical scheme makes it imperative to be able to write the equations describing compressible duct flow in the form of Eq. (4). 6 
The equations describing compressible flow can indeed be arranged as in Eq. (4), as described in Sec. V.

Sumirarizing, an implicit numerical scheme is used to solve for the pressure correction at each node. The iterative process continues until the

pressure correction $\tilde{\Delta p}$ approaches zero and the system is balanced. The numerical scheme is altered slightly by using the equation of state for a node that represents a volume or some capacitance. Thus, altering the modeling of flow in the elements or branches to account for compressible flow effects such as inertia or choking requires a linearized form for flow similar to Eq. (4).

\section{Far-Field Modeling Requirements}

Having reviewed the basic formulations and numerical scheme used in TVENT and recalling items listed in Ref. 2 that must be considered in modeling explosive phenomena, we now proceed with development of the far-field explosion code. To use TVENT as a basis for the explosion computer code requires the following modifications.

- Addition of the equation of state

- Calculation of system densities

- Calculation of system mass flows

- Addition of the energy equation

- Calculation of system temperatures

- Addition of the momentum equation, including inertia

- Calculation of system velocities

- Addition of compressible choking effects with dissipation

- Altering boundary conditions to accept parametric explosion parameters

- Input modification to accept additional information

- Altering output to nhtain mass flow, temperature, density, etc.

The features listed above were incorporated in developing the first version of a far-field explosion code. Other major steps involve many debugging runs of the computer code and exercising most of the features of the code on sample problems. Details of these steps and sample problems are described in the sections that follow. Also note that experiments must be performed to 
verify the inertia and choking effects. Experimental data are also needed on blower response to explosive transients. Little is known about this type of blower response, and it is not included in this far-field explosion code; therefore caution should be exercised in analyzing large pressure transients imposed across elements containing blowers.

D. Explosive Event Description

In this far-field analysis, the explosive event requires some form of simulation where the detail of the event is of no significance. Basically, an explosion can be defined by a rapid pressure rise, sometimes along with a rapid temperature rise. These pressure and temperature increases can result from physical, chemical, or even nuclear processes; for example, the rupture of a highly pressurized vessel (physical), the combustion or detonation of explosive materials (chemical), or criticality excursion of fissionable nuclear materials (nuclear) can cause a rapid pressure rise with or without a corresponding rapid temperature rise. All these processes involve a rapid mass and energy addition to a system, whether the system is closed or open. Analyses of explosions with rapid mass and energy additions are common and give good results if sufficient rate information is available. These approaches are useful for simulating an explosion inside a system and need detailed mass and energy source terms in the mass and energy equations. When the energy release rate is not known, we can use other information such as pressure-time or temperature-time profiles at a particular location in combination with mass addition information. The last two approaches require experimental data on the system that can be difficult to obtain. For an explosion outside a system, the pressure and temperature-time profiles can provide information needed to investigate a system response.

\section{MASS EQUATION}

As noted, the continuity equation must be applied at each node. In TVENT, where only incompressible flow is considered, a volumetric flow form can be used. To model compressible flows, we must account for density variations rather than simply balancing volumetric flows. Therefore, we must modify our continuity conditions in the explosion code at each node to include density 
and use a mass equation strictly for balancing. The mass equation for nodal points that have no mass storage is

$$
\sum_{k} q_{k} \dot{m}_{k}=\sum_{k} q_{k} \rho_{k} Q_{k}=0,
$$

where $\dot{m}_{k}$ and $Q_{k}$ are the mass flow rate and volumetric flow rate in branch $k$, and $\rho_{k}$ is the air density in branch $k$. The value $q_{k}$ is used to adjust for the proper flow direction in relation to the node; $q_{k}=+1$ for the downstream node of a branch or -1 for the upstream node.

The mass equation for nodal points that allow mass accumulation (capacitance nodes) is

$$
v \frac{d \rho}{d t}=\sum_{k} q_{k} \dot{m}_{k}+\dot{M}_{s}
$$

where $\dot{M}_{S}$ is the user-specified mass source per unit time for the volume and $V$ is the volume of the node. We use the ideal gas equation of state

$$
\rho=\frac{p}{R T},
$$

where $\mathrm{p}, \mathrm{T}$, and $\mathrm{R}$ are the node pressure, temperature, and gas constant for air. Putting the equation of state into Eq. (6) gives

$$
\frac{V}{R} \frac{d}{d t}\left(\frac{p}{T}\right)=\sum_{k} q_{k} \dot{m}_{k}+\dot{M}_{s} .
$$

Expanding and rearranging this equation gives

$$
\frac{d p}{d t}=\frac{R T}{V} \sum_{k} q_{k} \dot{m}_{k}+\frac{R T}{V} \dot{M}_{s}+R \rho \frac{d T}{d t}
$$


As in TVENT [see Ref. 1, Eq. $(E-6)], \frac{d p}{d t}$ is replaced by

$$
\frac{p^{n+1}-p^{n}}{\Delta t}=\frac{\tilde{p}+\tilde{\Delta p}-p^{n}}{\Delta t}
$$

with superscripts $\mathrm{n}+1$ and $\mathrm{n}$ indicating the new time and old time. $\widetilde{\Delta p}$ is the pressure correction term and $p$ the current pressure. The equation for $\widetilde{\Delta p}$ is [from Eq. (8)]

$$
\widetilde{\Delta p}=\left(p^{n}-\tilde{p}\right)+\frac{\Delta t R T}{V} \quad \sum_{k} q_{k} \dot{m}_{k}+\frac{\Delta t R T}{V} \dot{M}_{S}+\Delta t R \rho \frac{d T}{d t}
$$

The mass equation is implemented in two forms, depending on whether the room temperature is known (user-specified room condition) or unknown.

\section{A. Temperature Specified}

As in TVENT we can express the flow rate in terms of pressure [Ref. I, Eq. $(E-8)$ or Eq. (4)].

$$
\sum_{k} q_{k} \dot{m}_{k}=\sum_{k} q_{k} \rho_{k} Q_{k}=\sum_{k} q_{k}\left(\tilde{A}_{k}-\tilde{c}_{k} \tilde{\Delta p}\right) .
$$

Using Eq. (6) in Eq. (5) and solving for $\Delta p$ gives

$$
\tilde{\Delta p}=\frac{\left(p^{n}-\tilde{p}\right)+\frac{\Delta t R T}{V} \sum_{k} q_{k} \tilde{A}_{k}+\frac{\Delta t R T}{V} \dot{M}_{S}+\Delta t R \rho \frac{d T}{d t}}{1+\frac{\Delta t R T}{V} \sum_{k} q_{k} \tilde{C}_{k}} .
$$

The pressure correction term can be calculated explicitly from Eq. (12) by evaluating the other unknown quantities at the old time.

\section{B. Temperature Unknown}

When the temperature is not known, the temperature derivative term is eliminated from the mass equation [Eq. (10)] by evaluating it from the energy equation. 


\section{ENERGY EQUATION}

Both the amount and rate of energy release from an explosive event can have profound effects on the gas dynamics of a system. This becomes apparent when we recall that the pressure correction term $\Delta p$ derived in Sec. III is dependent on both temperature and time-rate of change of temperature. By maintaining an energy balance similar to the mass balance discussed in Sec. III, we can calculate the necessary system temperatures. The energy equation used in the far-field version of the explosion code with its corresponding effect on both nodal temperatures and the pressure correction term is outlined below.

\section{A. Arbitrary Node}

The basic energy equation employed is

$$
v \frac{d}{d t}(\overline{\rho u})=\sum_{k} q_{k} \dot{m}_{k}\left(u_{k}+\frac{P_{k}}{\rho_{k}}+\frac{v_{k}^{2}}{2}\right)+\dot{M}_{s} h_{s}+\dot{E}_{s},
$$

where $\overline{o u}$ represents the internal energy per unit volume and $u_{k}$ is the specific internal energy of the branch. The enthalpy associated with the mass addition is denoted by $h_{S}$, and $\dot{E}_{S}$ is an arbitrary energy source term. We make the additional assumption that

$$
\overline{\rho u}=\rho \bar{u},
$$

where $\rho$ is the average density in the volume and $\bar{u}$ the average specific internal energy. Then Eq. (13) becomes

$$
v \rho \frac{d \bar{u}}{d t}+\sqrt{u} \frac{d \rho}{d t}=\sum_{k} q_{k} \dot{m}_{k}\left(u_{k}+\frac{p_{k}}{\rho_{k}}+\frac{v_{k}^{2}}{2}\right)+\dot{M}_{s} h_{s}+\dot{E}_{s} .
$$


Putting Eq. (6) into this expression gives

$$
v \frac{d \bar{u}}{d t}=\sum_{k} q_{k} \dot{m}_{k}\left(u_{k}-\bar{u}+\frac{p_{k}}{\rho_{k}}+\frac{v_{k}^{2}}{2}\right)+\dot{M}_{s}\left(h_{s}-\bar{u}\right)+\dot{E}_{s} .
$$

Assuming a calorically perfect gas, the following relations hold.

$$
\begin{aligned}
& \bar{u}=C_{v}\left(T-T_{0}\right), \\
& C_{p}=C_{v}+R,
\end{aligned}
$$

and

$$
\frac{P}{\rho}=R T
$$

and $T_{0}, C_{p}$, and $C_{v}$ represent the reference temperature for internal energy, specific heats at constant pressure, and specific heats at constant volume. Using these expressions, Eq. (16) may be written.

$$
C_{v} v_{\rho} \frac{d T}{d t}=C_{p} \sum_{k} q_{k} \dot{m}_{k}\left(T_{k}+\frac{v_{k}^{2}}{{ }^{2} C_{p}}\right)-C_{v} T \sum_{k} q_{k} \dot{m}_{k}+\dot{M}_{s}\left(C_{p} T_{s}-C_{v} T\right)+\dot{E}_{s} .
$$

We can substitute Eq. (18) into Eq. (12) to obtain the pressure correction term. Or we can derive it directly from Eq. (13) because

$$
\overline{\rho U}=C_{v} \rho T=\frac{C_{v}}{R} p .
$$

So Eq. (13) becomes

$$
\frac{d p}{d t}=\frac{R}{C_{v} V}\left[\sum_{k} q_{k} \dot{m}_{k}\left(C_{p} T_{k}+\frac{v_{k}^{2}}{2}\right)+\dot{M}_{s} C_{p} T_{s}+\dot{E}_{s}\right]
$$


and after some algebraic manipulations, the pressure correction term is

$$
\begin{gathered}
\tilde{\Delta p}=\left\{\left(p^{n}-\tilde{p}\right)+\frac{\gamma R \Delta t}{V}\left[\sum_{k} q_{k} \tilde{A}_{k}\left(T_{k}+\frac{v_{k}^{2}}{2 C_{p}}\right)+\dot{M}_{s} T_{s}+\frac{\dot{E}_{s}}{C_{p}}\right]\right\} \\
/\left[1+\frac{\gamma R \Delta t}{V} \sum_{k} q_{k} \tilde{C}_{k}\left(T_{k}+\frac{v_{k}^{2}}{2 C_{p}}\right)\right]
\end{gathered}
$$

with $T_{s}$ being the temperature associated with the mass addition.

\section{B. Noncapacitance Nodes}

For a zero-volume (noncapacitance) node, the energy equation takes on a simple form. Setting $V=0, \dot{E}_{S}=0$, and $\dot{M}_{S}=0$ in Eq. (18) gives

$$
c_{p} \sum_{k} q_{k} \dot{m}_{k}\left(T_{k}+\frac{v_{k}^{2}}{2 C_{p}}\right)-c_{v} T \sum_{k} q_{k} \dot{m}_{k}=0 \text {. }
$$

But by the mass equation for noncapacitance nodes, Eq. (5), the second term is zero and the equation becomes

$$
c_{p} \sum_{k} q_{k} \dot{m}_{k}\left(T_{k}+\frac{v_{k}^{2}}{2 C_{p}}\right)=0 \text {. }
$$

To calculate the new nodal temperature, we divide the branch flows into two groups: one flowing into the node, another out of the node. The latter group will have the temperature of the node. The final result for the nodal temperature is

$$
T=\left\{\left[\sum_{k} q_{k} \dot{m}_{k}\left(T_{k}+\frac{v_{k}^{2}}{2 C_{p}}\right)\right]_{\text {in }}+\left[\sum_{k} q_{k} \dot{m}_{k} \frac{v_{k}^{2}}{2 C_{p}}\right]_{\text {out }}\right\} /\left[-\sum q_{k} \dot{m}_{k}\right]_{\text {out }}
$$


Once the temperature is known, density is calculated from the equation of state.

V. MOMENTUM EQUATION WITH INERTIA

A. Motivation

The momentum equation used in the TVENT code is basically quasi-steady; it is an orifice flow relationship that is adequate for slow-flow transients such as the type induced by a tornado. However, in the case of an explosion, the pressure rise can be quite rapid and the effect of inertia must be taken into account, no matter how small it may seem to be. The development and implementation of the momentum equation with inertia into the explosion code should make it useful in a much broader range of far-field problems.

B. Development of Equation

We start with the momentum equation in differential form for one-dimensional, constant-area, compressible, unsteady adiabatic gas flow with friction. $3^{3}$

$$
\frac{\partial}{\partial t}(\rho v A)+\frac{\partial}{\partial x}\left(\rho v^{2} A\right)=-\frac{\partial}{\partial x}(p A)-\frac{f}{D} \frac{\rho v|v|}{2} A
$$

where $t$ and $x$ are time and space coordinates; $\rho, v, A, p, f$, and $D$ represent density, velocity, flow area, pressure, Darcy friction factor, and hydraulic diameter, respectively. The last term on the right side of the equation is a loss term where loss is due to wall friction. Defining mass flow rate $\mathrm{m}$,

$\dot{m}=\rho \vee A$,

Eq. (25) can be rewritten as

$$
\frac{1}{A} \frac{\partial}{\partial t}(\dot{m})+\frac{\partial}{\partial x}\left(\frac{\dot{m}^{2}}{\rho A^{2}}\right)=-\frac{\partial}{\partial x} p-\frac{f}{D} \frac{\dot{m}|\dot{m}|}{2 \rho A^{2}} .
$$



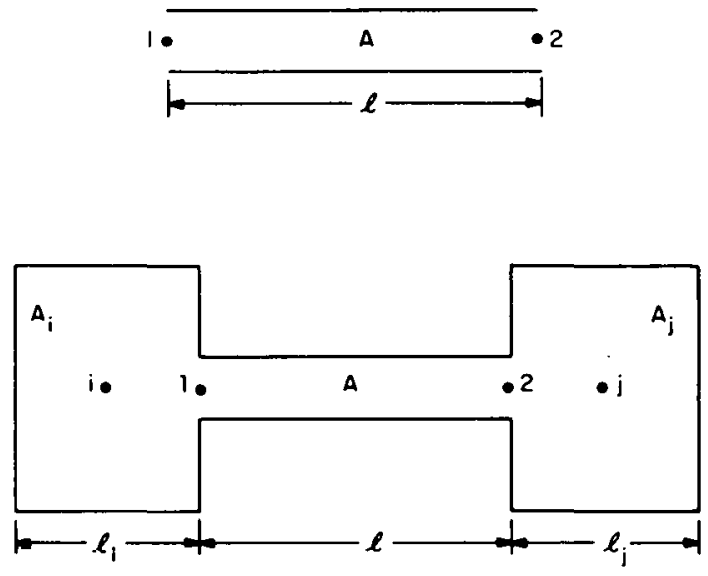

Fig. 3. Branch with sudden area change.

Integration along a duct with length $\ell$ (Fig. 3) yields:

$$
\frac{l}{A} \frac{\overline{d m}}{d t}+\frac{1}{A^{2}}\left(\frac{\dot{m}^{2}}{\rho_{2}}-\frac{\dot{m}^{2}}{\rho_{1}}\right)=-\left(p_{2}-p_{1}\right)-\frac{f}{D} \frac{1}{2 A^{2}} \frac{\dot{m}|\dot{m}|}{\rho} .
$$

Quantities with - indicate average values over the length. Because a proper density should be selected for the branch, we shall make a further approximation in this aspect later. The partial differential with respect to time is replaced by the total differential in subsequent relationships.

Tn accommodate a more general configuration, such as two rooms connected by a duct, the flow through each room $c$ an be modeled the same way as a duct (Fig. 3). The combined one-dimensional flow path is from $i$ to 1,1 to 2 , and 2 to $j$, with finite area change at interfaces 1 and 2 . The interface continuity relations are:

$$
\left.\dot{m}_{i}\right|_{1}=\left.\dot{m}\right|_{1},\left.\quad \dot{m}_{j}\right|_{2}=\left.\dot{m}\right|_{2} \text {. }
$$

The subscripts 1 and 2 indicate the interface locations; $m_{j}, m$, and $m_{j}$ are the mass flow rates for each leg of the branch. 
Application of this momentum equation is limited to the far-field region, and the flow remains subsonic, although choking is allowed. A step further, we assume the spatial variation of the mass flow rate is negligible,

$$
\frac{\partial \dot{m}}{\partial x}=0
$$

Also, we assume the difference in kinetic energy at both ends is negligible,

$$
\left(\frac{\dot{m}^{2}}{\rho}\right)_{2}-\left(\frac{.2}{\mathrm{~m}}\right)_{1}=0 \text {, }
$$

which leads to a momentum equation of incompressible. form from Eq. (28),

$$
\frac{\ell}{A} \frac{d \dot{m}}{d t}=-\left(p_{2}-p_{1}\right)-\frac{f}{D} \frac{1}{A^{2}} \frac{\dot{m}|\dot{m}|}{2 p} \ldots
$$

The - associated with $\dot{m}$ can be dropped. Adding the momentum equations for the three legs and using the interface continuity relations we obtain

$$
I \frac{d \dot{m}}{d t}=\left(p_{i}-p_{j}\right)-K_{e f f} \frac{1}{A^{2}} \frac{\dot{m}|\dot{m}|}{2 \dot{p}},
$$

where

$$
\begin{aligned}
& I=\frac{l_{i}}{2 A_{i}}+\frac{\ell}{A}+\frac{l_{j}}{2 A_{j}} \text {, and } \\
& K_{\text {eff }}=\left(\frac{f l_{j}}{2 D_{i}}+k_{i}\right)\left(\frac{A}{A_{j}}\right)^{2}+\frac{f l}{D}+k+\left(\frac{{ }^{f} l_{j}}{2 D_{j}}+k_{j}\right)\left(\frac{A}{A_{j}}\right)^{2} .
\end{aligned}
$$

I represents the inertia effect of the flow path, including the rooms as well as the duct. Keff is the total effective resistance coefficient; the minor losses, such as turning, entrance, and exit are represented by the $K$ 's: The first and the third terms in Eq. (35) are usually quite small because the area 
ratios are squared and can be neglected. Notice that only half the room characteristic length $\ell$ in the flow direction is used in the inertia and friction calculations for that branch because the other half contributes the same to the branch on the other side of the room. This is consistent with the onedimensional approach for a pipe divided into a number of control volumes. For a room with only one branch, the other half. will be considered as a stagnant (no-flow) region. For a pure node (room with zero volume), there is no effect on the inertia or friction, as seen in Eqs. (34) and (35). Note that I and $K_{\text {eff }}$ are input quantities.

\section{Numerical Procedure}

Although Eq. (33) can be integrated readily to obtain the mass flow-rate, the difficulty lies in the fact that the pressure differential term is not known. In fact, it is the eigenvalue of the integration determined by the mass and the energy conservations at the nodes or in the rooms.

In finite-difference form Eq. (33) becomes:

$$
\frac{I}{\Delta t}\left(\dot{m}^{n+1}-\dot{m}^{n}\right)=\left(p_{i}^{n+1}-p_{j}^{n+1}\right)-k_{e f f} \frac{1}{A^{2}} \frac{\dot{m}^{n+1} \mid \dot{m}^{n+1}}{2 p}
$$

Superscripts $n$ and $n+1$ indicate the value at time $n \Delta t$ and $(n+1) \Delta t$. This finite-difference scheme is used to avoid the singularity as the inertia coefficient I becomes zero. Rewriting Eq. (36) and introducing s gives

$$
\frac{s k_{e f f}}{2 A^{2 p}}\left(\dot{m}^{n+1}\right)^{2}+\frac{I}{\Delta t} \dot{m}^{n+1}+\left(p_{j}^{n+1}-p_{i}^{n+1}\right)-\frac{I}{\Delta t} \dot{m}^{n}=0
$$

where $s=1$ if $\dot{m}^{n+1} \geq 0$, and $s=-1$ if $\dot{m}^{n+1}<0$.

The positive flow direction is selected according to the steady-state solution. Equation (37) can be solved by using the quadratic equation formula. This well-known approach has been used in some reactor containment analysis codes. 4 


$$
\dot{m}^{n+1}=\frac{1}{2 a s}\left(-b \pm \sqrt{b^{2}-4 a s c}\right) \text {, }
$$

with

$$
\begin{aligned}
& a=\frac{k_{\text {eff }}}{2 A^{2} \rho}, \\
& b=\frac{I}{\Delta t},
\end{aligned}
$$

and

$$
c=\left(p_{j}^{n+1}-p_{i}^{n+1}\right)-\frac{I}{\Delta t} \dot{m}^{n}
$$

The positive sign is needed in Eq. (39) for realistic solution; $s$ is selected to maintain -4 asc $>0$ at all times.

Eq. (39) is incomplete because the pressure differential term through $c$ is not yet known. The mass and energy flow rates must satisfy conservation requirements at nodes and rooms. Following the iteration process used in the TVENT code, Eq. (39) can be put in a perturbation form similar to Eq. (4):

$$
\dot{m}=\tilde{A}-\tilde{C} \Delta \tilde{p}
$$

Here, we drop superscript $n+1$ but add to indicate temporary value. $\Delta p$ represents the pressure perturbation at a certain node $j$; its vanishing value means convergence is reached. For a small perturbation, we substitute

$$
p_{j}=\tilde{p}_{j}+\tilde{\Delta p}
$$


or

$$
c=\tilde{c}+\tilde{\Delta p}
$$

into Eq. (39) and obtain

$$
\tilde{A}=\frac{\tilde{B}^{1 / 2}-b}{2 \text { as }}
$$

and

$$
\tilde{C}=\frac{q}{\widetilde{B}^{I / 2}} \text {, }
$$

with

$$
\tilde{B}=b^{2}-4 a s c
$$

and

$$
\tilde{c}=\left(\tilde{p}_{j}-\tilde{p}_{i}\right)-\frac{I}{\Delta t} \dot{m}^{n} .
$$

Again, quantities with are adjusted at each iteration. q takes the value of +1 if the pressure correction is performed on the downstream node; otherwise it is -1 . The amount of pressure correction for a node is determined by the mass conservation equation

$$
\sum_{k} q_{k} \dot{m}_{k}=0 \text {. }
$$


Combining Eqs. (41) and (44) gives

$$
\widetilde{\Delta p}=\frac{\sum_{k} a_{k} \tilde{A}_{k}}{\sum_{k} a_{k} \tilde{c}_{k}} .
$$

We also note that positive $q_{k} \tilde{A}_{k}$ means that branch $k$ feeds mass into the node $j$ or vice versa. The above iteration is repeated until the magnitude of the pressure correction is less than a given convergence factor. Of course, the pressure correction is carried out at all nodes simultaneously throughout the field except at the boundary nodes. The pressure adjustment for a capacitance node (room) is done in a slightly different manner as we shall see next.

We emphasized earlier that in the case of an explosion, temperature variations can be quite large, and the isothermal approximation can introduce significant error, even in a single time step during which the pressure iteration is carried out. In TVENT, the equation relating the pressure change in a room with volume $V$ to the density change $[$ see $\mathrm{Eq} .(6)]$ is

$$
V \frac{d p}{d t}=V R T \frac{d \rho}{d t}=R T \sum_{k} q_{k} \dot{m}_{k}
$$

The first equality is only an approximation. Combining the continuity, energy, and equation of state, we can obtain the equation for the pressure change [see Eq. (20)].

$$
\frac{d p}{d t}=\frac{R \gamma}{v} \sum_{k} q_{k} \dot{m}_{k}\left(T_{k}+\frac{v_{k}^{2}}{2 C_{p}}\right) .
$$

$R, r$, and $C_{p}$ are the gas constant, specific heat ratio, and specific heat at constant pressure. $T_{k}$ and $U_{k}$ represent the branch temperature and velocity. Kinetic energy of the branch is included in this expression. The 
finite-difference form of Eq. (47) after combining with the iteration expression for $p$ yields

$$
\widetilde{\Delta p}=\left[c_{f} \sum_{k} q_{k} \tilde{A}_{k}\left(T_{k}+\frac{v_{k}^{2}}{2 C_{p}}\right)+(p-\tilde{p})\right] /\left[1+c_{f} \sum_{k} q_{k} \tilde{c}_{k}\left(T_{k}+\frac{v_{k}^{2}}{2 C_{p}}\right)\right] \text {, }
$$

where

$$
C_{f}=\frac{\gamma R \Delta t}{V},
$$

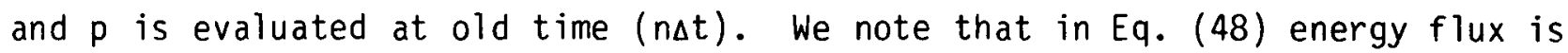
needed for pressure correction in a room. In the case of a room with external mass and energy addition to simulate an explosion condition, the first set of square brackets can include some additional terms on their effects.

So far, we have seen the development of the momentum equation with inertia and the limitation in its application, as well as some refinement in the pressure correction in a room. As the flow velocity increases, a point called choked flow is reached where the flow rate is no longer determined by the momentum equation. The choking flow calculations are discussed in Sec. VI.

D. Branch Properties

The momentum equation establishes the relation between pressure drop and flow rate. Density, a constant for incompressible flow, is the only flow parameter required to complete the description of the condition. In the case of compressible flow, the incompressible formulation is still adequate as long as the flow speed is small compared with the speed. of sound and the duct length is not so long (compared to a typical wavelength) as to cause substantial pressure drop. For steady-state and low-speed flow, the common practice of averaging the upstream and downstream densities to calculate the pressure differential is acceptable. However, at high-speed flow, the branch density can be quite different from the downstream node if it is a room or a boundary. The use of the upwind property in many fluid-mechanical problems is widely accepted. The upstream node density for the branch used at high-speed flow is still adequate if the duct is short; the same argument can be applied to the branch temperature. 
The above discussion can be readily incorporated into the calculative scheme if the upstream node is a zero-volume node. The matter becomes more complicated if it is a boundary node or a room. For strictly one-dimensional flow, the temperature in a room is related to the branch upstream node by the energy equation

$$
T_{i}+\frac{v_{i}^{2}}{2 C_{p}}=T_{1}+\frac{v_{1}^{2}}{2 C_{p}},
$$

and from the continuity equation

$$
\rho_{i} v_{i} \quad A_{i}=\rho_{1} v_{1} A
$$

Subscript $i$ indicates the upstream node condition. A is the branch flow area, and the characteristic flow area for the room is represented by $A_{i}$. For $a$ boundary, $A_{i}$ is a very large number, so the kinetic energy term can be ignored and $T_{i}$ is the stagnation temperature of the node. The difference between $T_{1}$ and $T_{j}$ is small if the flow velocity is small.

One more equation is needed to solve for the three unknowns in Eqs.

and (51). Section V.B discusses the one-dimensional assumption that the flow area should be constant. Equation (33) is an equivalent formulation of this condition for the configuration shown in Fig. 3. In fact, the equivalent branch length for inertia, from Eq. (34), is

$$
\ell_{e f f}=\ell+\ell_{i} \frac{A}{2 A_{i}}+\ell_{j} \frac{A}{2 A_{j}}
$$

The equivalent upstream node condition is assumed to relate to the actual node condition by the isentropic processes,

$$
\frac{\rho_{1}}{\rho_{1}}=\left(\frac{T_{1}}{T_{i}}\right)^{\frac{1}{\gamma-1}}
$$


and $\frac{p_{1}}{P_{i}}=\left(\frac{T_{1}}{T_{i}}\right)^{\frac{\gamma}{\gamma-1}}$,

because we have already combined all the losses (friction and minor) of the room into the $\mathrm{K}_{\text {eff }}$, Eq. (35), which belong to the branch downstream of this node. Equations (50)--(53a) can be solved numerically, and then the equivalent upstream node pressure can be calculated by Eq. (53b). There is no similar pressure calculation for the downstream end of the branch because the pressure should be the same as the nodal pressure according to the theory of jet dissipation. 5

Finally, the pressure differential term in Eq. (36) should be adjusted if the upstream node is a room or boundary. Throughout the pressure iteration process, the nodal temperature is allowed to vary, as well as the branch temperature and density, as in Eqs. (50) and (51).

\section{CHOKING OF COMPRESSIBLE FLOW WITH DISSIPATION}

\section{A. Motivation}

The steady-state flow rate in incompressible flow is determined by the pressure drop. In compressible flow, the flow rate will reach a maximum value regardless of how much the downstream pressure is decreased if the upstream pressure is constant. This phenomenon is called choking. In a ventilation system with tornado-induced pressure trdusients, choking is not expected to occur, but in an explosion, large pressure differentials will exist at some nodal points. Hence, we must use the proper flow rate for a branch under choking conditions. For isentropic (adiabatic, frictionless) flow, choking can be easily established and the maximum flow rate is uniquely determined by the upstream condition. This is no longer a simple matter when dissipation is present.

\section{B. Critical Upstream Mach Number}

We will investigate the quasi-steady compressible flow inside a constantarea duct, where the usual one-dimensional approximation is assumed. Heat transfer is not allowed but friction effect is present. In general, this duct 
will connect two rooms (Fig. 3) as shown in the momentum equation development. Only subsonic flow will be considered.

Assuming a constant upstream condition, the flow rate increases as the downstream pressure decreases, and at the same time, the Mach number at the duct exit (location 2) increases. Eventually, the downstream pressure becomes critical and further reduction of the downstream pressure will not create any increase in flow rate because the Mach number at location 2 has reached unity. Therefore, no disturbance signal can transmit through this point from downstream to upstream by wave propagation, and the flow rate or any flow properties upstream of this point cannot be adjusted to suit the new downstream condition. At this moment, the Mach number at the duct entrance (location 1) reaches a maximum also, but the value is less than 1 . This upstream critical Mach number $M_{1}$ is uniquely related to the friction loss by the relation ${ }^{3}$

$$
K=\frac{1-M_{1}^{2}}{\gamma M_{1}^{2}}+\frac{\gamma+1}{2 \gamma} \ln \left[\frac{(\gamma+1) M_{1}^{2}}{2\left(1+\frac{\gamma-1}{2} M_{1}^{2}\right)}\right]
$$

where $\gamma$ is the specific heat ratio and $K$ is the resistance coefficient, including the wall friction and entrance losses. The exit loss does not enter into this relation because it is caused by the loss of kinetic energy formed by a jet downstream of location $2^{5}$. Once we know the friction loss, the critical upstream Mach number can be calculated by one numerical iteration. Without the friction loss $(K=0)$, the Mach number at location 1 becomes unity. This is the same conclusion for an ideal orifice or a convergent nozzle.

\section{Critical Mass Flow Rate}

As soon as the critical upstream Mach number is known, the maximum mass flow rate $c$ an be calculated if the pressure and density at location 1 are known. Using the definition of Mach number

$$
M_{1}=v_{1} / c_{1} \text {, }
$$


where $v_{1}$ and $c_{1}$ are the local gas velocity and local speed of sound, which in turn is a function of pressure $p_{1}$ and density $p_{1}$, with

$$
c_{1}=\sqrt{r p_{1} / \rho_{1}}
$$

for an ideal gas, we have

$$
\dot{m}=\rho_{1} v_{1} A=A M_{1} \sqrt{\gamma p_{1}^{\rho} \rho_{1}}
$$

This is the maximum allowable mass flow rate that a particular. branch can supply for a given condition at 1 . We emphasize the importance of using $p_{1}$ and $\rho_{1}$. For a true node, $p_{1}$ and $\rho_{1}$ represent the actual nodal values. If the upstream node is a room (or boundary) as seen in Fig. $3, p_{1}$ and $\rho_{1}$ are not the same as $\mathrm{p}_{i}$ and $\rho_{j}$-- in fact they are related to the upstream room conditions as described in Sec. V.D. For a room with a very large flow cross section and with some algebraic manipulation,

$$
\dot{m} \frac{\dot{A}}{\frac{r}{R} \frac{p_{1}}{T_{i}} M_{1}}\left[1+\frac{r-1}{2} M_{1}^{2}\right]^{\frac{1}{2}},
$$

which is the familiar expression for the ideal orifice flow rate if the stagnation temperature $T_{i}$, the local pressure, and the local Mach number are knnwn. 3

D. Numerical Procedure

Equation (57) is similar to the equation for incompressible orifice flow, except that the pressure differential is replaced by the upstream pressure only. The pressure correction can be achieved by introducing a small perturbation quantity $\Delta p$ and we have

$$
\dot{m}=\tilde{A}-\tilde{C} \tilde{\Delta p}
$$

where

$$
\tilde{A}=s M_{1} \sqrt{r p_{1} \rho_{1}}
$$


and

$$
\widetilde{C}=-\frac{\tilde{A}}{2 p_{1}} .
$$

Al1 quantities with are the updated values during the iteration process. $M_{1}$ is constant because it is a unique function of the friction loss. Equation (59) fits the numerical scheme used previously in TVENT. Because the mass flow rate depends on the upstream pressure only, and if a node is downstream of a branch through which the choking occurs, no pressure correction should be made on that node by this branch. In this case, the pressure adjustment coefficient $C$ must be set equal to zero. That condition is determined by the combined effect of $s$ and $q$ defined in Sec. $V$.

\section{VESSEL DISCHARGE AND CHARGING VESSEL PROBLEMS}

Some experimental data in Ref. 6 describe the discharge of high-pressure gas (air) from a vessel to the atmosphere. This reference also discusses the pressurization of a vessel by a high-pressure air supply reservoir. These are interesting test cases for our computer code, especially in the areas of mass and energy conservation, orifice flow relation and choked flow. In both cases, shown schematically in Fig. 4, the initial pressure differential is quite large, and the flow is choked during the early phase of the transient. As the vessel pressure approaches that of ambience or of the supply reservoir, the unchoked orifice flow relationship applies. Although the unchoked orifice relation is essentially an incompressible formulation, the choked flow calculation does, include the effect of dissipation. 3

The experimental and analytical pressure transients and all pertinent parameters are shown in Figs. 5 and 6 . For the numerical calculation, the dimensionless resistance coefficient is first estimated based on some typical orifice information, and then the dimensional resistance coefficient is calculated, because the latter is the required input used in the code. The figures show that the analytical result compares well with the experiment, even though there is some uncertainty about the orifice resistance. The transition from choked flow to unchoked flow is best illustrated in the charging vessel case (Fig. 6). The constant mass and energy supply from an 


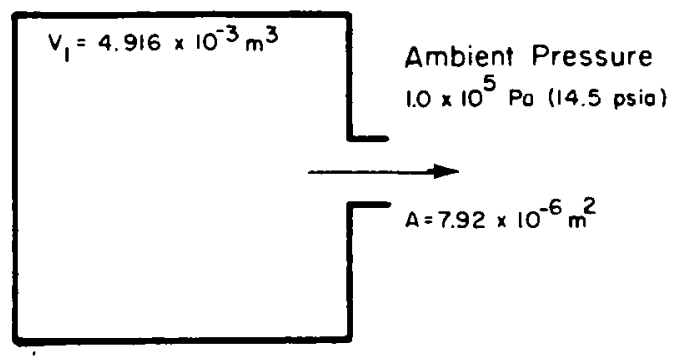

Initial Pressure: $\quad 1.479 \times 10^{6} \mathrm{Po}(214.5 \mathrm{psia})$

Initial Temperature: $\quad 294\left(69^{\circ} \mathrm{F}\right)$

(a) Discharging Vessel

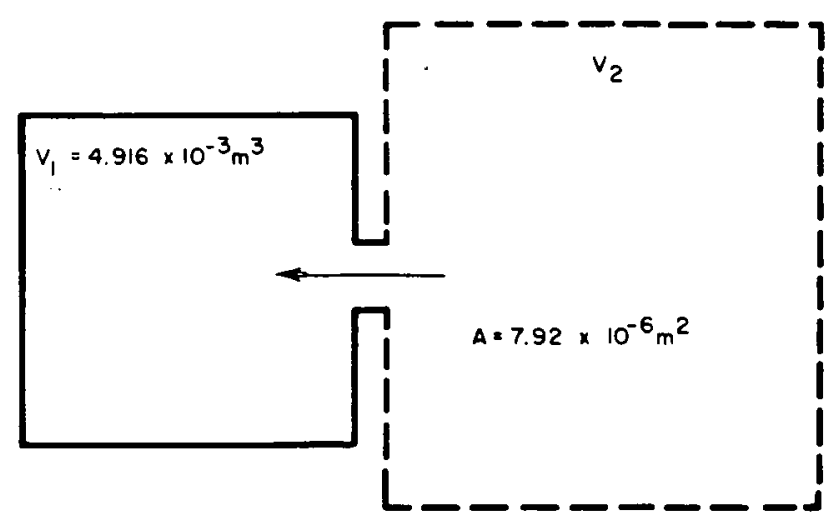

Fig. 4. Schematic and conditions for charging and discharging problems.

Initiol Pressure: $1.0 \times 10^{5} \mathrm{~Pa}(14.5 \mathrm{psio})$

Initial Temperature: $294 \mathrm{~K}\left(69^{\circ} \mathrm{F}\right)$

Supply Pressure: $6.83 \times 10^{5} \mathrm{Po}$ (99 psia)

$v_{2}>>v_{1}$

(b) Charging Vessel

infinite rescrvoir throughout the rhoking phase yields a constant slope in the pressure transient curve. The pressure rise eventually levels off because the decreasing mass and energy flow rate depend on the pressure differential between the vessel and the reservoir. The vessel discharge has a similar transition from choking to nonchoking, but it is not as easy to detect by the pressure transient alone because the choked flow depends on the charging vessel condition as well as the unchoked flow. These results show that our explosion code predicts these relatively simple cases quite well. We will test the code for other cases as experimental data become available. Also, we plan some simple experiments that include effects of inertia. 


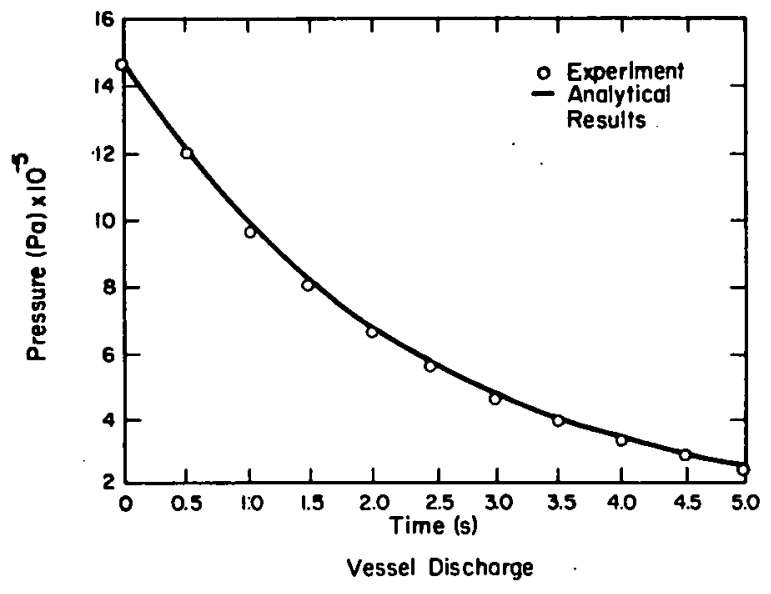

Fig. 5. Analytical and experimental results for discharging problem.

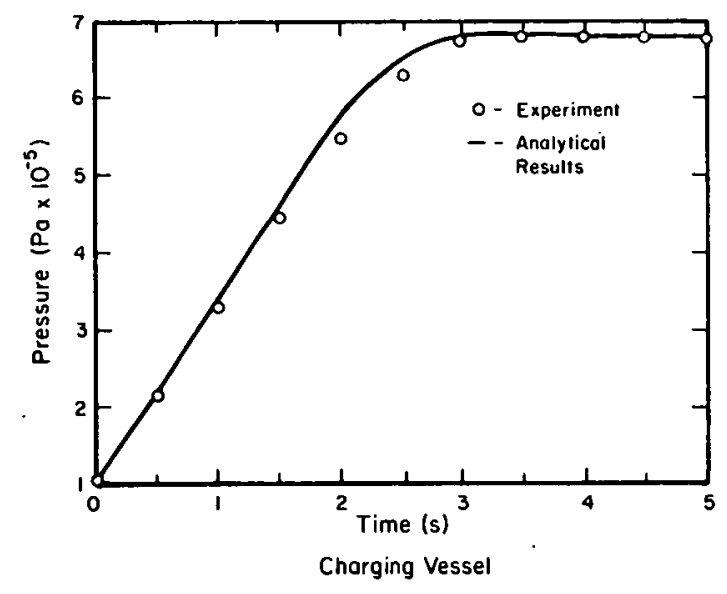

Fig. 6. Analytical and experimental results for charging problem.

VIII. EXAMPLE PROBLEMS USING THE EXPLOSION CODE

The problems discussed in Sec. VI illustrate the capability of the computer code to model effects of mass and energy conservation, the orifice flow relation used, and choking. However, other types of problems are better suited to demonstrate the code's capability to model simulated explosions.

\section{A. Modeling Considerations}

Use of our explosion code for modeling requires more information than that required for TVENT. For example, the flow algorithms for inertia and choking effects are based on the properties of the ducts, and therefore detailed duct dimensions are required in those parts of the system where inertia and choking are to be modeled. In general, the lumping of elements in the model can be done as coarsely as in TVENT, but a capability exists to allow subdivision of the network elements into finer zones.

An explosion can be modeled in several ways; the options differ only in the manner of specifying the energy of the explosion. The more direct approach is to specify the injection of mass and the energy associated with an explosion. An indirect approach is to specify the injection of mass, but to express energy in terms of a pressure or temperature rise. Theoretically, either approach is 
valid. However, the option used will depend on the information available; that is, the lack of good detail about the explosion might determine which option to use. The use of the indirect approach with the room temperature $r$ ise is the most demanding because the shape of the temperature rise function is used to determine the time derivative of temperature.

\section{B. Example Problems}

The first example problem demonstrates options that are available for specifying an explosion. We have also analyzed this model for a tornado-pressure transient with both TVENT and the explosion code to provide a comparison. The second sample problem emphasizes the importance of using volume distribution or element subdivision in obtaining detailed distribution of results.

Although the primary purpose of these sample problems is to demonstrate the capability of the explosion code, they also show some things to consider in preparing the required input. The selection of these examples was arbitrary, and no consideration was given to simulation of actual facilities.

1. Example 1. This problem is taken from the existing waste calcining facility ventilation system at the Idaho Chemical Processing Plant (ICPP) in Idaho Falls, Idaho. This facility was used to test TVENT during its development stage and results were reported. 7

The original TVENT model, modified to account for explosive effects, was poorly defined because there was little information about the older ICPP structures. That information is still not available, but the problem was chosen because it is relatively simple and has been analyzed with TVENT.

The model used in our explosion code is shown in Fig. 7. The explosion is applied to the small room at node No. 7. It was not applied to nodes 3 or 4 because explosion effects would have been greatly diminished in these large volumes. The node 7 location could simulate an explosion in a glovebox near. the exhaust of the large room at node 4. A filter was added to the system simply to include all types of elements. The model is terminated at node 11.

This sample problem shows four equivalent options available for specifying an explosion. This problem is also a comparison of TVENT and our explosion code for a tornado transient. 
2. Example 2. This fictitious problem was designed to provide detailed information on the effects of inertia, choked flow, and subdivision of a duct. The system consists of an explosion chamber connected to a long pipe that has a constant area of $0.0729 \mathrm{~m}^{2}\left(0.785 \mathrm{ft}^{2}\right)$. A typical smooth-pipe friction drop has been assumed for calculating the branch resistances. This problem has no initial flow and so must be handled as a restart problem. The explosion code model used for this problem is shown in Fig. 8. The pipe has been broken arbitrarily, into eight segments to provide additional detailed information along the length of the duct. Two explosion magnitudes are used--a highintensity explosion and a low-intensity explosion. These levels were chosen arbitrarily for demonstration. The high yield is equivalent to a $378 \mathrm{~kg} / \mathrm{s}$ $\left(3.0 \times 10^{6} \mathrm{lb} / \mathrm{hr}\right)$ mass injection at a temperature of $538^{\circ} \mathrm{C}\left(1000^{\circ} \mathrm{F}\right)$ for a duration of $0.1 \mathrm{~s}$. The lower intensity is the same except that mass injection has been reduced by a factor of 10 .

\section{Results}

The explosion code output formats are the same as those generated by TVENT except that temperature has been added to the list of parameters. A comment statement has been added to the table called "Problem Summary of Extreme Values," to indicate the occurrence of choking. The plotting formats used in this report were made by a program called DISSPLA. The values of pressures, flow, and temperature are given in standard output units; that is, inches of water, cfm, and degrees F. Equivalent SI unit values are not shown because the only purpose is comparison of the codes.

1. Example Problem 1. The four options are based on different ways of expressing the energy of the explosion as indicated by the numbers in parentheses following the title, Explosion in Glovebox, shown in Figs. 9--11.

$\left(\begin{array}{llll}0 & 1 & 0 & 0\end{array}\right)$ = temperature of injected mass specified,

$\left(\begin{array}{llll}1 & 2 & 0 & 0\end{array}\right)$ = energy of injected mass specified,

$\left(\begin{array}{llll}0 & 2 & 1 & 0\end{array}\right)$ = pressure transferred to confined air specified, and

$\left(\begin{array}{llll}0 & 2 & 0 & 3\end{array}\right)$ = temperature transferred to confined air specified. 


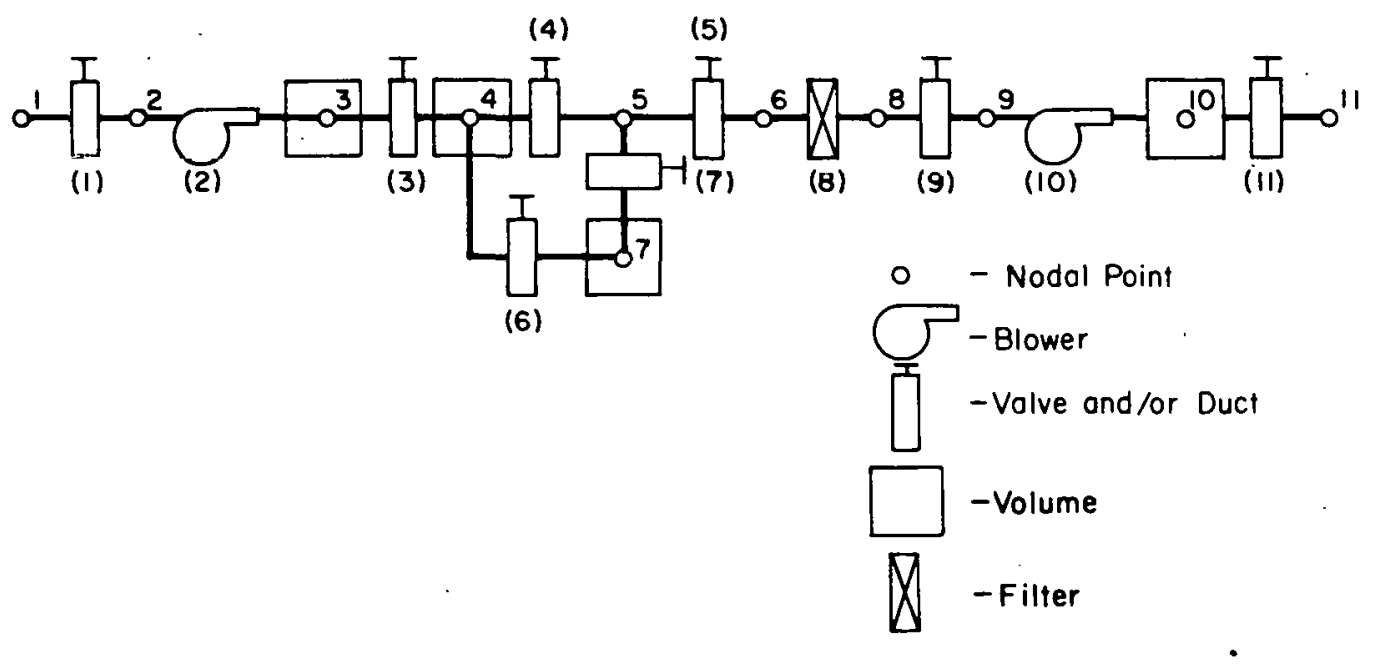

Fig. 7. Model ventilation system for example 1.

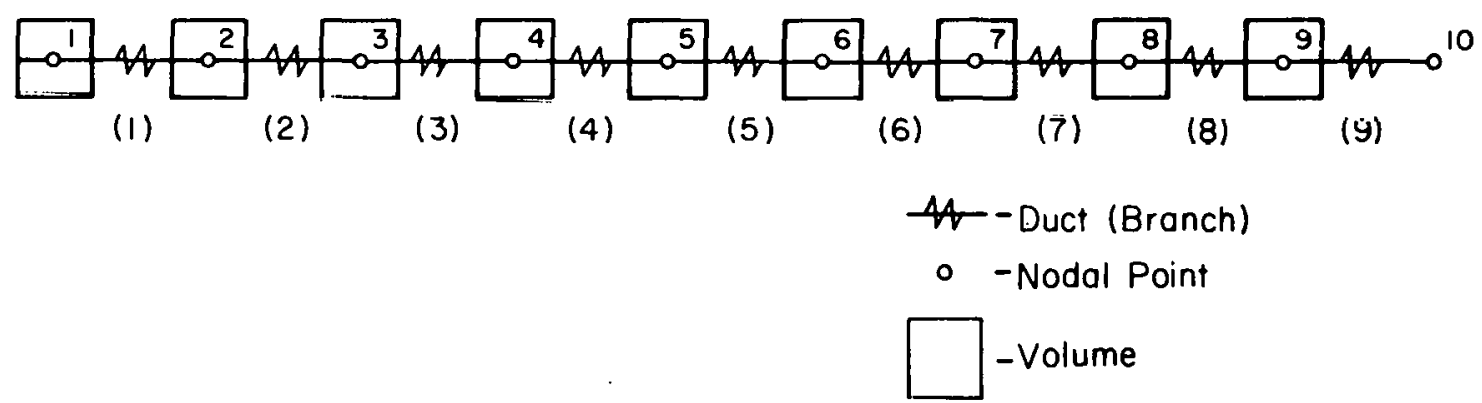

Fig. 8. Computer code model for cxample 2. 
The pressure and temperature values transferred from the explosion to the surrounding air in the room were obtained from the output of one of the first two option solutions. To ensure a well-defined temperature function (to avoid large slope discontinuities), 50 points were used with a smaller spacing in high gradients regions. The temperature and pressure build-up in the room containing the explosion are shown in Figs. 9 and 10 . Typical system volume flows are shown in Fig. 11 .

The results shown in Figs. 9--11 support the conjecture that all the available options for specifying an explosion are indeed equivalent. However, this will not always be the case, because in real situations the parameters will not all be known with this degree of accuracy.

Pressure and volume flow curves in Figs. 12 and 13 indicate no significant differences between the results of TVENT and our explosion code. This confirms the assumption that the simpler flow algorithms of TVENT were adequate to handle tornado-sized pressure transients, which results in a significant saving in computer time. Only minor differences occur in the comparison, especially in the volume flows. The computer running times are 2.688 and $6.683 \mathrm{~s}$, respectively, for TVENT and our explosion computer code.

2. Example Problem No. 2. Typical output parameter plots comparing the low- and high-intensity explosions are shown in Figs. 14 and 15.

Inertia and capacitance in a system cause a delay in the flow as it moves through a system. Friction causes a loss of energy resulting in a continuous decrease in flow values. The propagation of the pressure, temperature, and flow along the pipe shown in Figs. 14 and 15 confirms these effects. The increased flow at the end of the pipe (branches 8 and 9 in Fig. 14) is due to a decrease in density, which causes an increase in velocity to maintain a given mass flow.

The Summary of Extreme Values indicates that choked flow occurs at $0.7 \mathrm{~s}$ for the high-intensity explosion problem. This condition is also evident in the volume flow plot for branch 9 (Fig. 14) by a flattened curve beginning at $0.7 \mathrm{~s}$. This condition occurs when the Mach number reaches unity, and it means that the mass flow has reached a maximum value. 

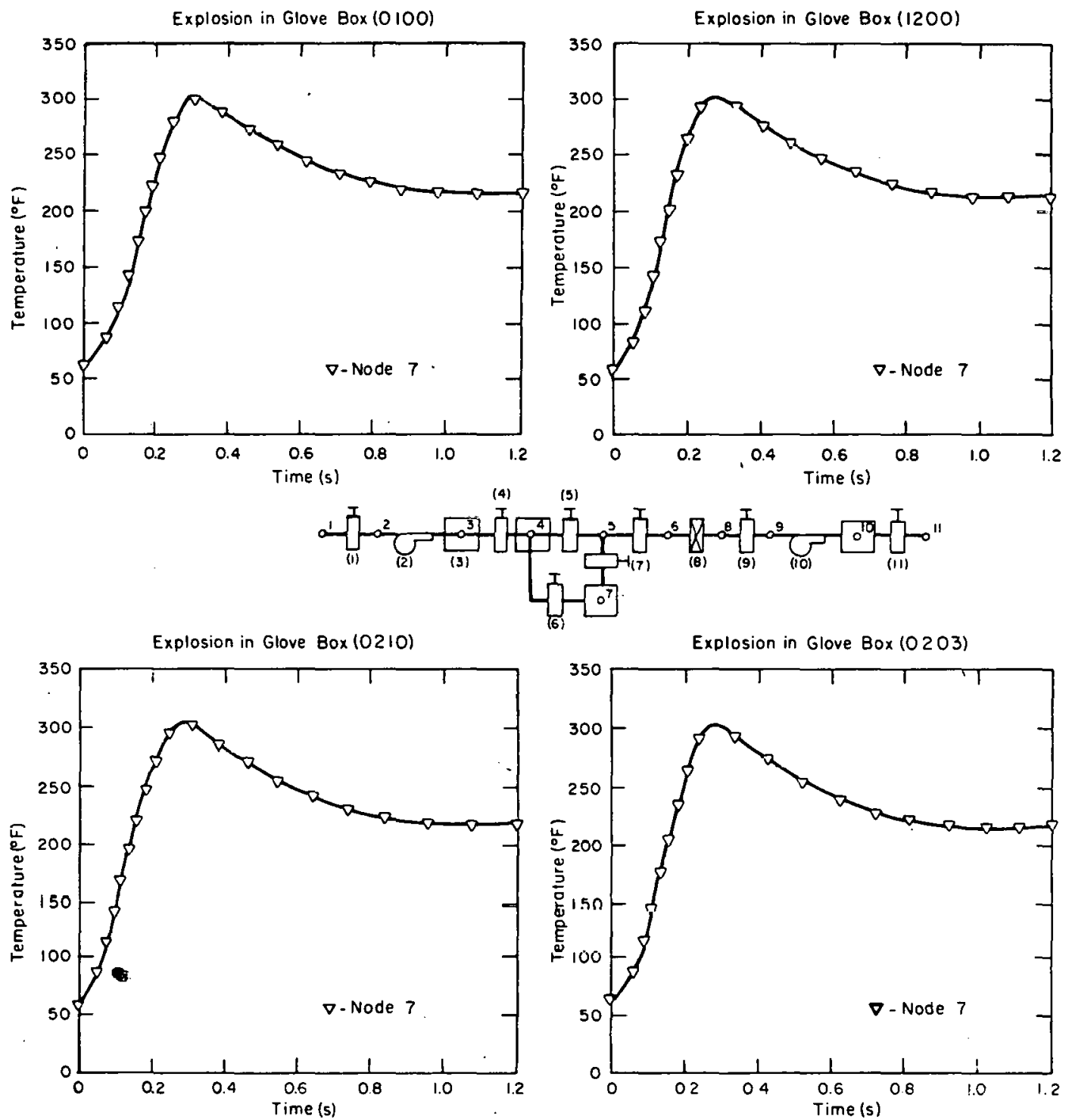

Fig. 9. Temperature $\left({ }^{\circ} \mathrm{F}\right)$ increase for explosion at node 7. 

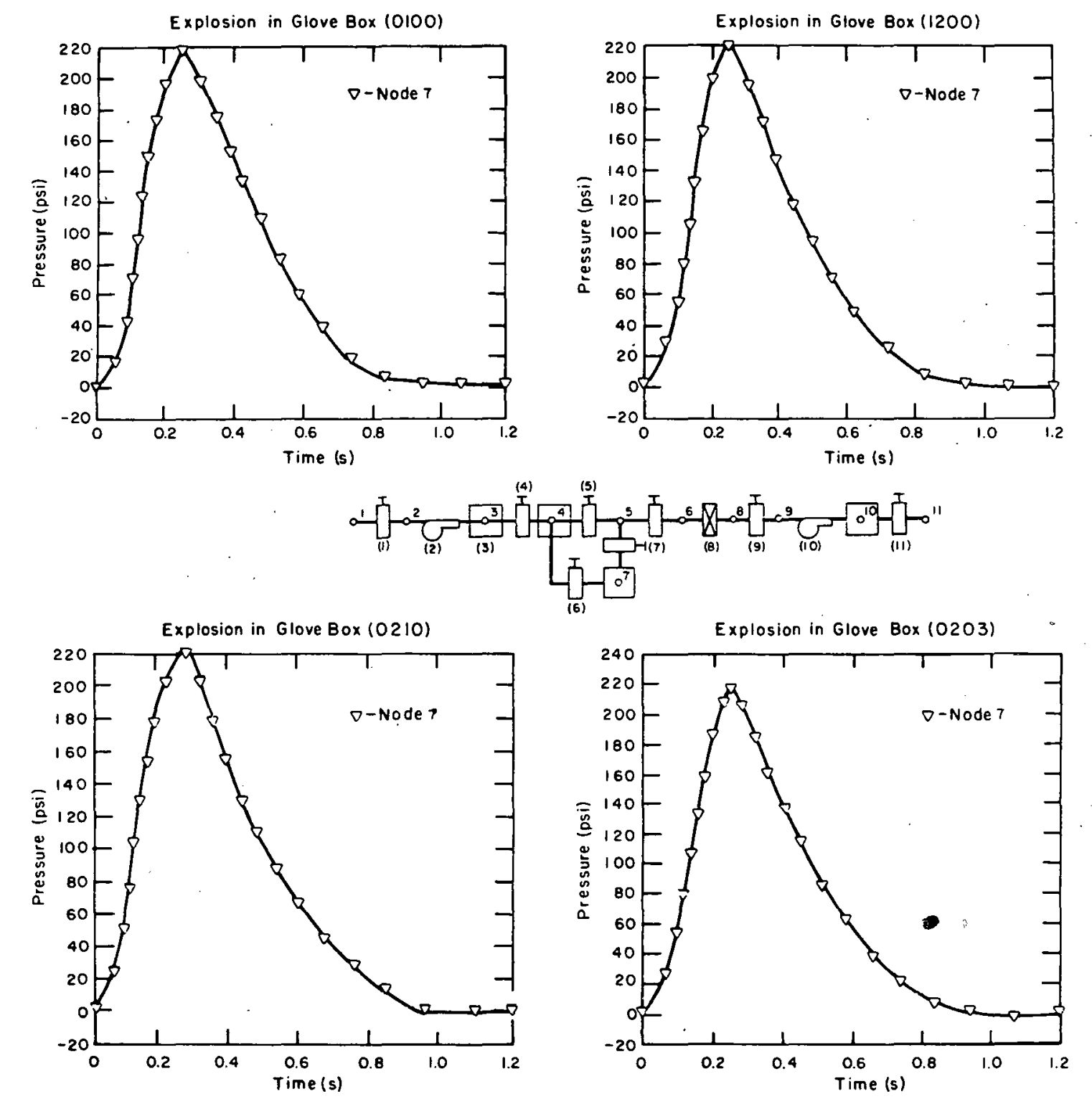

Fig. 10. Pressure (psig) increase for an explosion at node 7. 

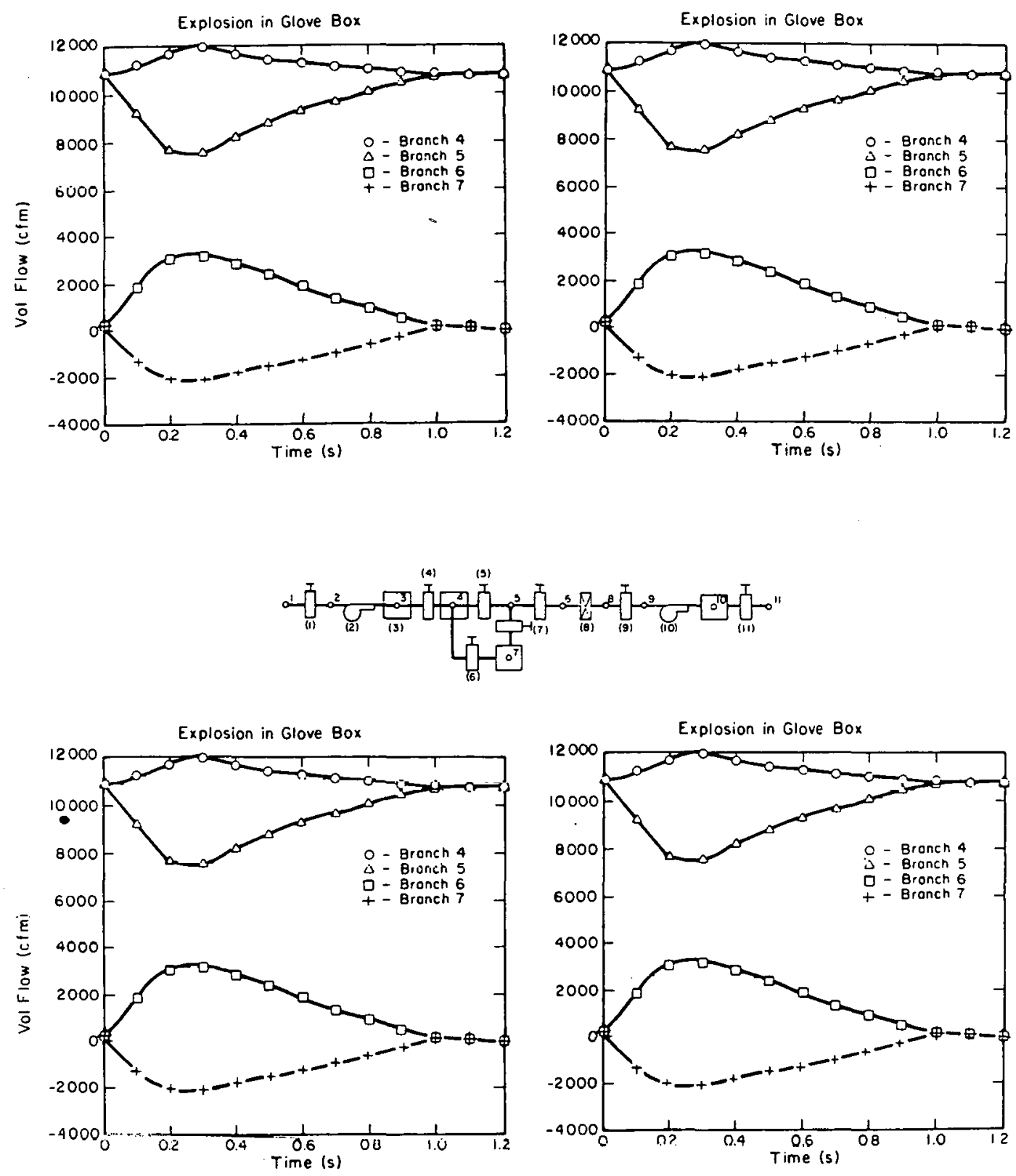

Fig. 11. System flows (cfm) for an explosion at node 7 . 

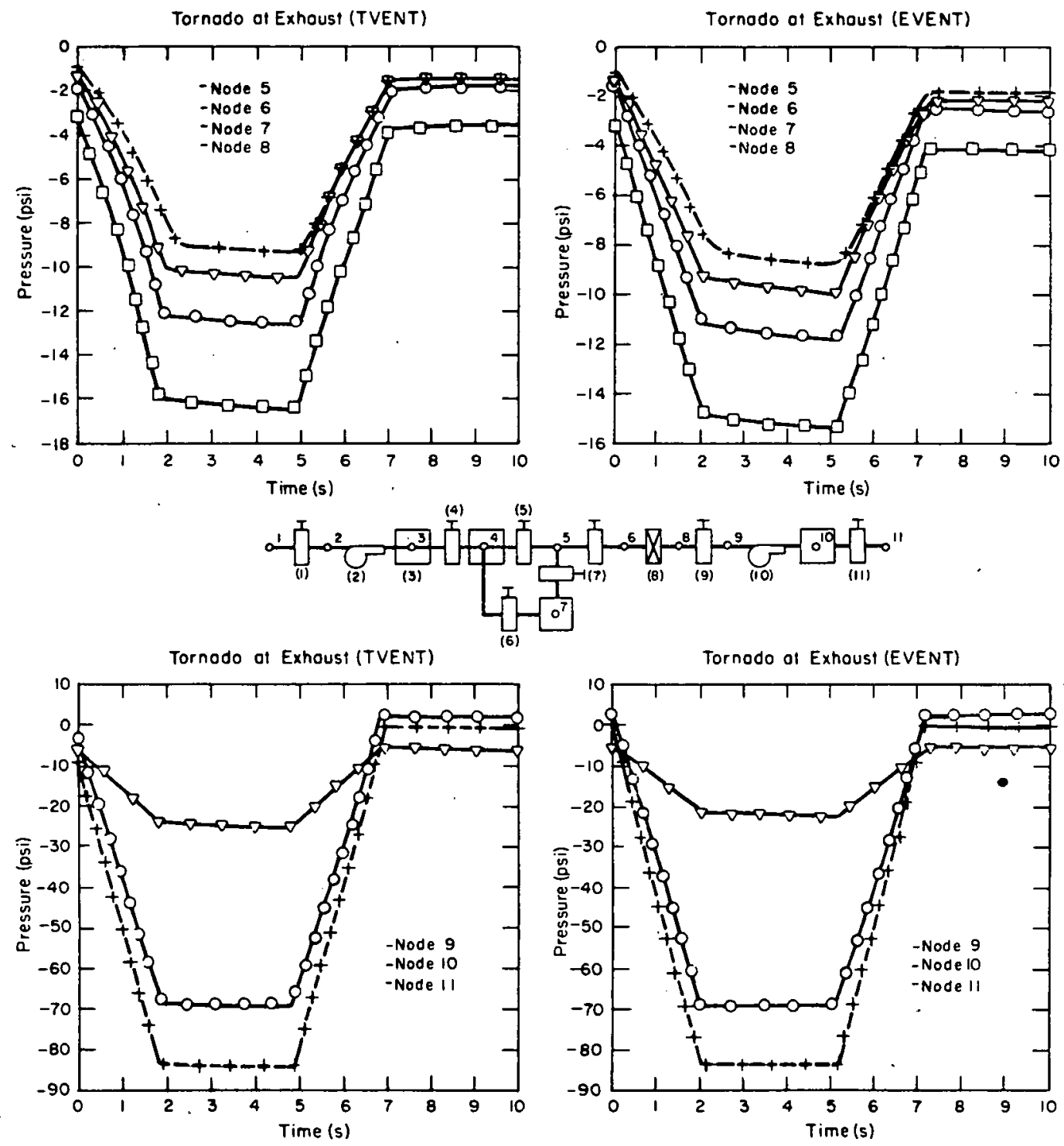

Fig. 12. Pressure transients for TVENT and the explosion computer code. 

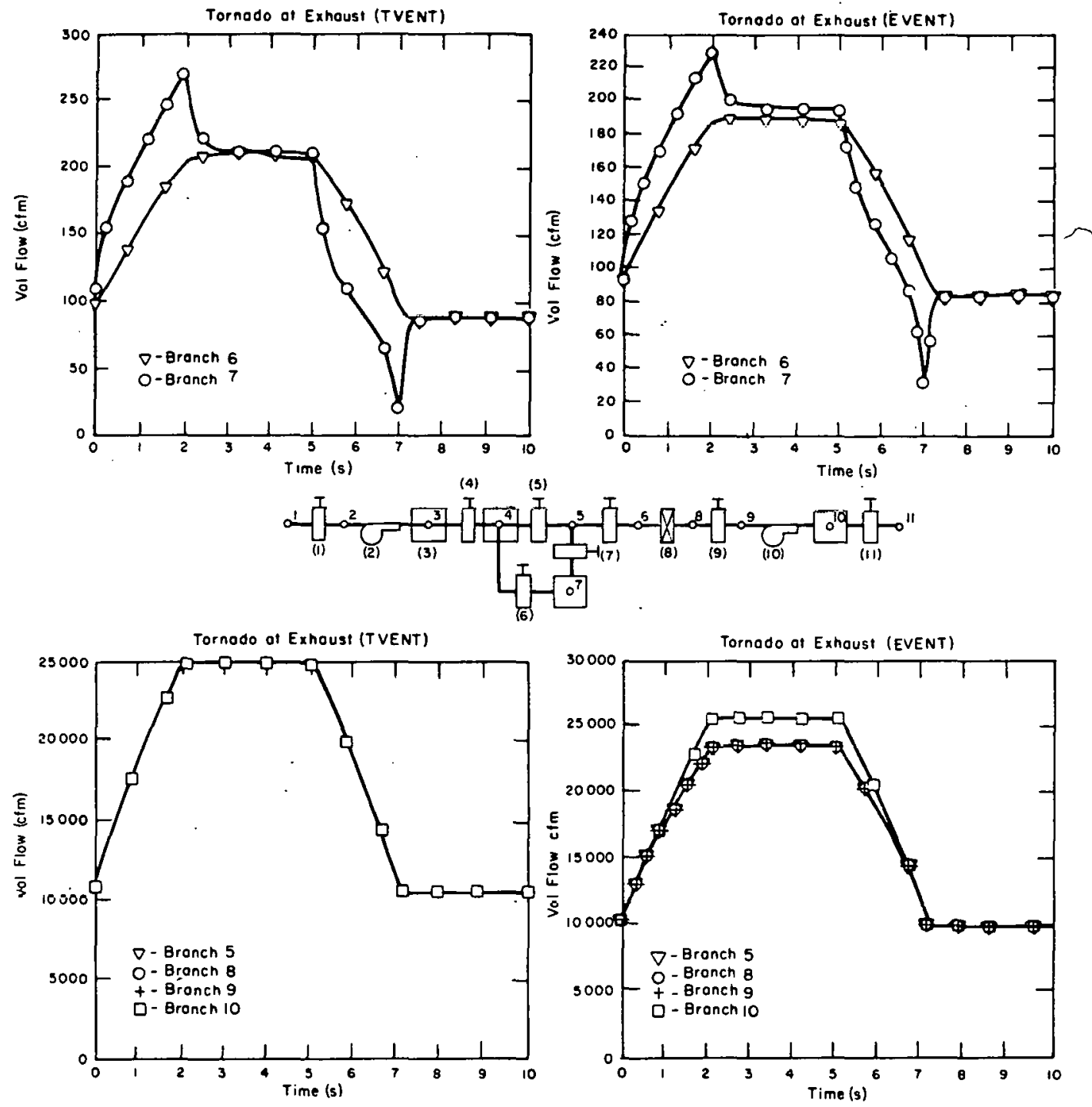

Fig. 13. Volumetric flow for TVENT and the explosion computer code. 

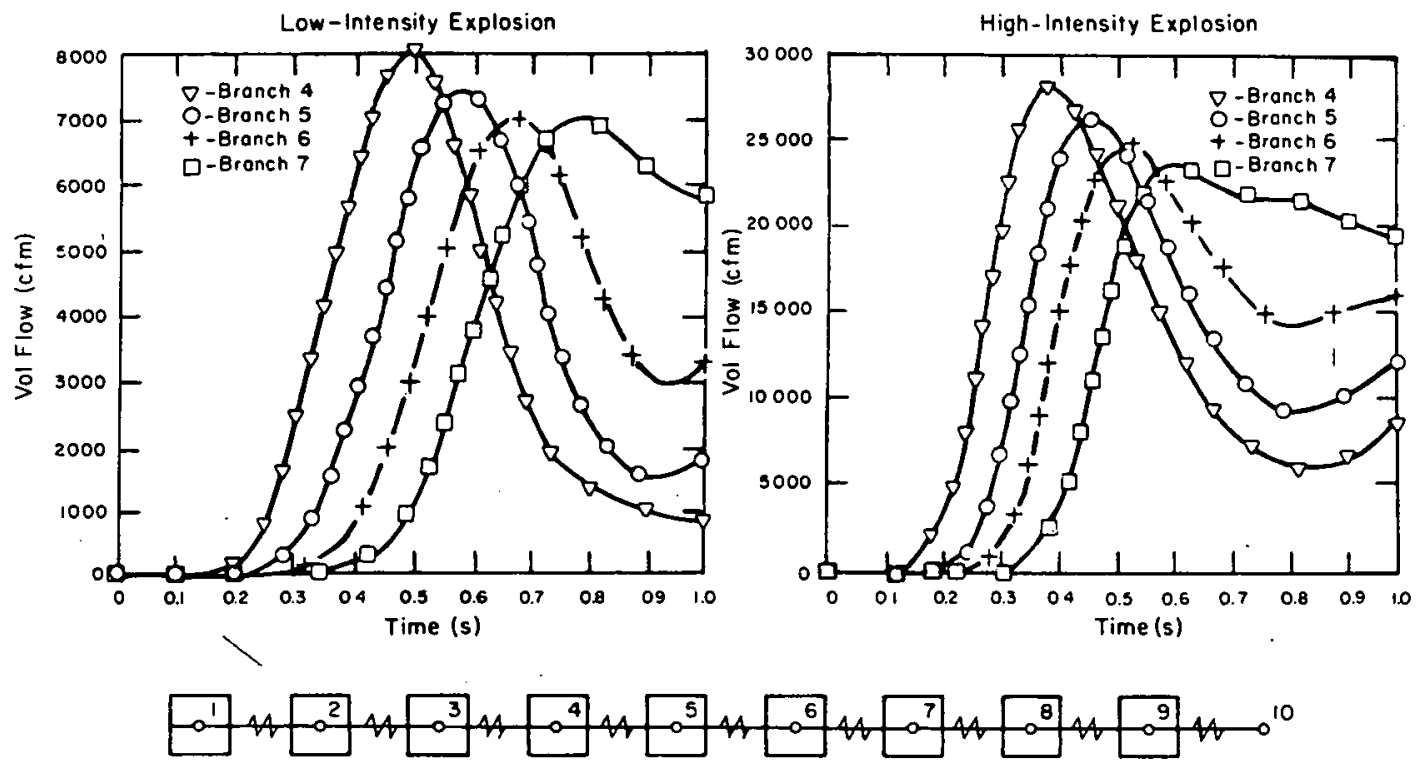

(1)

(2)

(3)

(4)

(5)

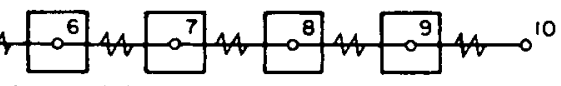

(6)

(7)

(8)

(9)
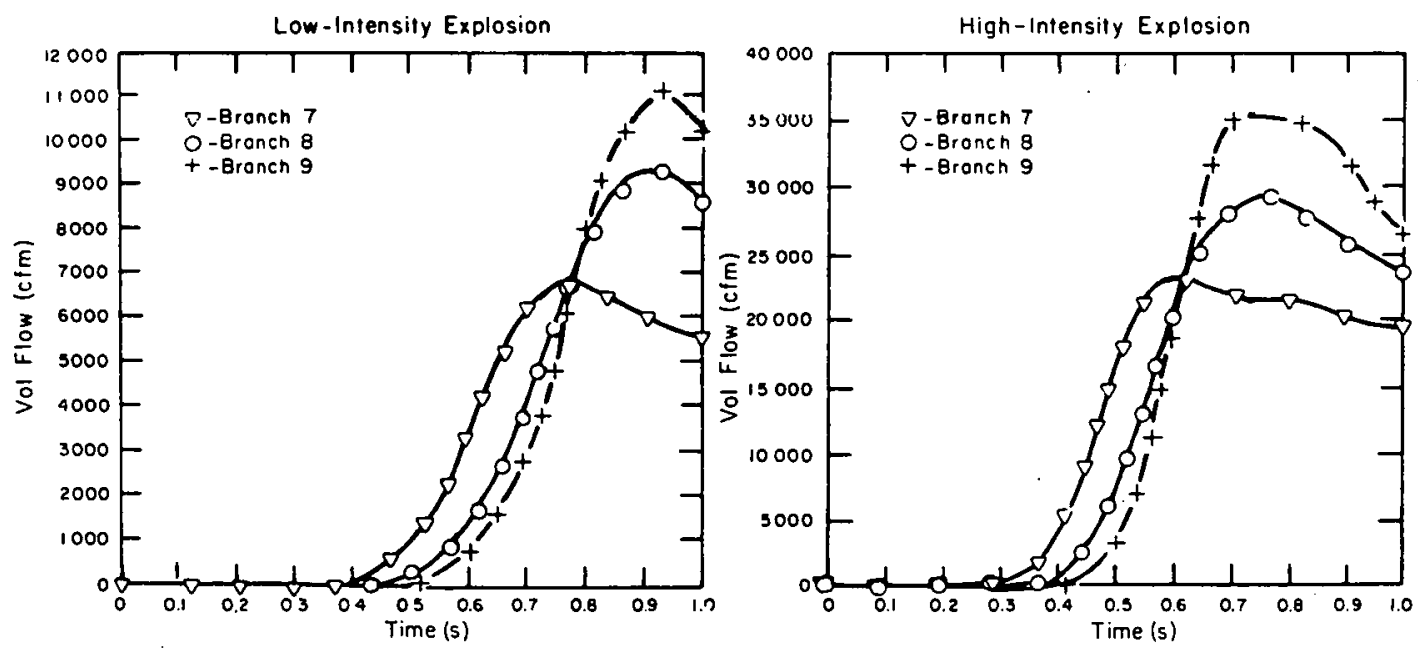

Fig. 14. Volumetric flows in a long duct for two explosion intensities. 

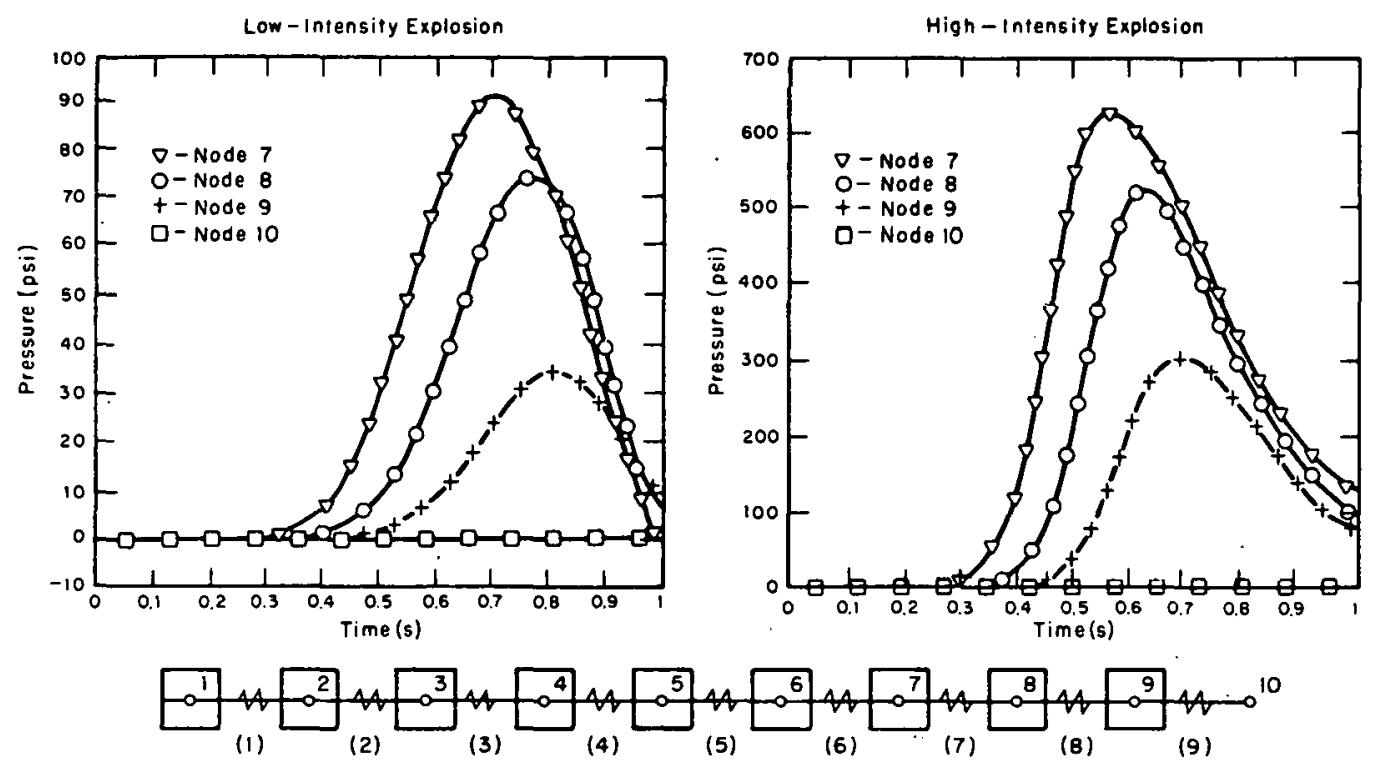

(1)

(2)

(3)

(4)

(6)

(B)

(9)

\& - Duct (Bronch)

o-Nodol Point

- volume

Low - Intensity Explosion

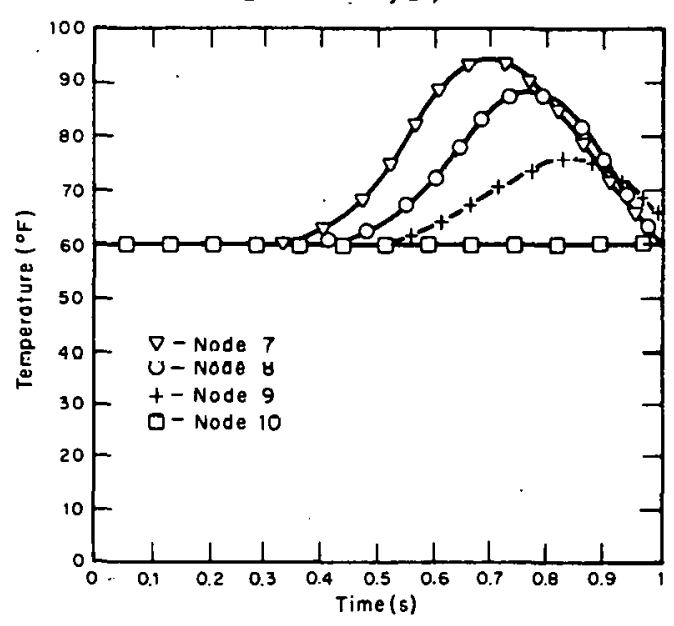

High - Intensity Explosion

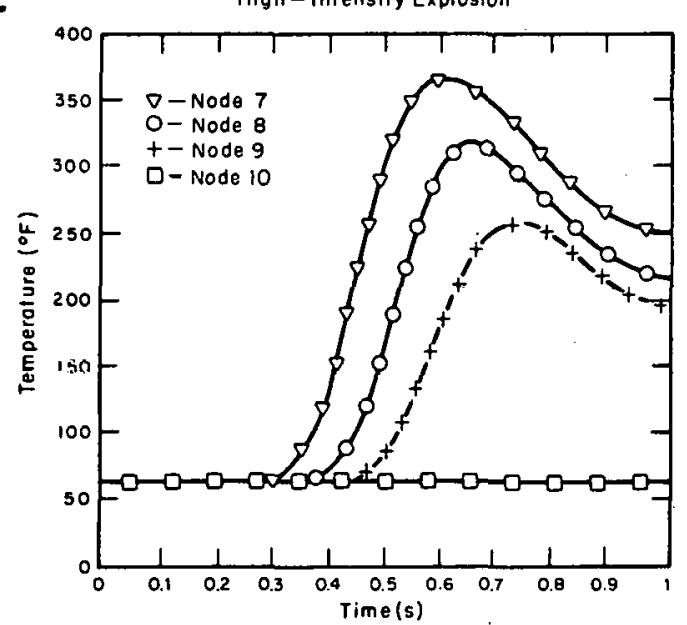

Fig. 15. Temperature and pressure distribution in a long duct for two explosion intensities. 
IX. STATUS

The computer code has been released in preliminary form to friendly users to allow it to be exercised. Their suggestions for improvement will be considered when we release the final version. These users will apply the code to the analyses of structures where explosive potentials exist. This will insure that the code can model realistic conditions and point out facility deficiencies that must be remedied.

We plan to release the code and documentation unconditionally to the $\mathrm{Na}-$ tional Energy Software Center at the Argonne National Laboratory by the end of FY 1980.

\section{$X$. SUMMARY}

We have described development of a first-version computer code that can be used to calculate explosively induced transients within the ventilation systems of nuclear facilities. This explosion code version is suitable for modeling far-field explosive effects; that is, it can be used to model pressure, density, and temperature fields that are somewhat remote from or relatively insensitive to the explosive event. This version of the code will be useful to obtain a first-order solution to a problem and indeed may be the only analysis required in many situations. This approach and future developments of the explosion computer code were outlined in Ref. 2 .

The code retains all the features of the TVENT code but uses a mass equation, an energy equation, and a momentum equation for calculating compressible flow throughout a network system. The numerical technique used in TVENT is preserved, and both inertia and choking effects are included. Example problems that demonstrate the capabilities and limitation of the code are described. 


\section{REFERENCES}

1. K. H. Duerre, R. W. Andrae, and W. S. Gregory, "TVENT - A Computer Program for Analys is of Tornado-Induced Transients in Ventilation Systems, "LoS Alamos Scientific Laboratory report LA-7397-M (July 1978).

2. W. S. Gregory, P. R. Smith, J. W. Bolstad, and K. H. Duerre, "Analys is of Ventilation Systems Subjected to Explosive Transients -- Initial Analysis and Proposed Approach," Los Alamos Scientific Laboratory report LA-7964-MS (August 1979).

3. A. H. Shapiro, The Dynamics and Thermodynamics of Compressible Fluid Flow (Ronald Press, New York, 1953).

4. R. G. Gido, C. I. Grimes, R. G. Lawton, and J. A. Kudrick, "COMPARE: A Computer Program for the Transient Calculation of System of Volumes Connected by Flowing Vents," Los Alamos Scientific Laboratory report LA-NUREG-6488-MS (September 1976).

5. V. Ingard and H. Ising, "Acoustic Nonlinearity of an Orifice," Journal of the Acoustic Society of America 42, 6-17 (1967).

6. A. L. Addy and B. J. Walker, "Rapid Discharging of a Vessel Through a Nozzle or an Orifice," ASME paper 72-FE-40 (1972).

7. W. S. Gregory, R. W. Andrae, K. H. Duerre, and R. C. Dove, "Ventilation System Analysis During Tornado Conditions," Los Alamos Scientific Laboratory report LA-6999-PR (October 1977). 

Los Alamos 